\title{
YAP1 oncogene is a context-specific driver for pancreatic ductal adenocarcinoma
}

\author{
Bo Tu, ${ }^{1}$ Jun Yao, ${ }^{1}$ Sammy Ferri-Borgogno, ${ }^{2,3}$ Jun Zhao, ${ }^{2}$ Shujuan Chen, ${ }^{1}$ Qiuyun Wang, ${ }^{1}$ Liang Yan, ${ }^{1}$ \\ Xin Zhou, ${ }^{1,4}$ Cihui Zhu, ${ }^{1}$ Seungmin Bang, ${ }^{5}$ Qing Chang, ${ }^{6}$ Christopher A. Bristow, ${ }^{6}$ Ya'an Kang, ${ }^{7}$ \\ Hongwu Zheng, ${ }^{8}$ Huamin Wang, ${ }^{2}$ Jason B. Fleming, ${ }^{7,9}$ Michael Kim, ${ }^{7}$ Timothy P. Heffernan, ${ }^{6}$ \\ Giulio F. Draetta, ${ }^{10}$ Duojia Pan, ${ }^{11}$ Anirban Maitra, ${ }^{2,3}$ Wantong Yao, ${ }^{10,12}$ Sonal Gupta, ${ }^{2,3}$ \\ and Haoqiang Ying ${ }^{1}$ \\ 'Molecular and Cellular Oncology Department, 2Pathology Department, and ${ }^{3}$ Sheikh Ahmed Center for Pancreatic Cancer \\ Research, The University of Texas MD Anderson Cancer Center, Houston, Texas, USA. ${ }^{4}$ Department of Obstetrics and \\ Cynecology, Shengjing Hospital, China Medical University, Shenyang, Liaoning, China. ${ }^{5}$ Department of Internal Medicine, \\ Institute of Gastroenterology, Yonsei University College of Medicine, Seoul, South Korea. ${ }^{6}$ Institute for Applied Cancer \\ Science and 'Surgical Oncology Department, The University of Texas MD Anderson Cancer Center, Houston, Texas, USA. \\ ${ }^{8}$ Department of Pathology and Laboratory Medicine, Weill Cornell Medical College, New York, New York, USA. ${ }^{9}$ Department \\ of Gastrointestinal Oncology, Moffitt Cancer Center, Tampa, Florida, USA. ${ }^{10} \mathrm{Cenomic}$ Medicine Department, The University \\ of Texas MD Anderson Cancer Center, Houston, Texas, USA. "Department of Physiology, Howard Hughes Medical Institute, \\ University of Texas Southwestern Medical Center, Dallas, Texas, USA. ${ }^{12}$ Translational Molecular Pathology Department, \\ The University of Texas MD Anderson Cancer Center, Houston, Texas, USA.
}

Transcriptomic profiling classifies pancreatic ductal adenocarcinoma (PDAC) into several molecular subtypes with distinctive histological and clinical characteristics. However, little is known about the molecular mechanisms that define each subtype and their correlation with clinical outcome. Mutant KRAS is the most prominent driver in PDAC, present in over $\mathbf{9 0 \%}$ of tumors, but the dependence of tumors on oncogenic KRAS signaling varies between subtypes. In particular, the squamous subtype is relatively independent of oncogenic KRAS signaling and typically displays much more aggressive clinical behavior versus the progenitor subtype. Here, we identified that yes-associated protein 1 (YAP1) activation is enriched in the squamous subtype and associated with poor prognosis. Activation of YAP1 in progenitor subtype cancer cells profoundly enhanced malignant phenotypes and transformed progenitor subtype cells into squamous subtype. Conversely, depletion of YAP1 specifically suppressed tumorigenicity of squamous subtype PDAC cells. Mechanistically, we uncovered a significant positive correlation between WNT5A expression and YAP1 activity in human PDAC and demonstrated that WNT5A overexpression led to YAP1 activation and recapitulated a YAP1-dependent but Kras-independent phenotype of tumor progression and maintenance. Thus, our study identifies YAP1 oncogene as a major driver of squamous subtype PDAC and uncovers the role of WNT5A in driving PDAC malignancy through activation of the YAP pathway.

Conflict of interest: GFD reports personal fees from and stock ownership in Karyopharm Therapeutics, Forma Therapeutics, Metabomed, BioveloclTA, Nurix, and Orionis Biosciences and personal fees from Blueprint Medicines, Taiho Pharmaceutical, Symphogen, and Helsinn Ventures.

Copyright: (c) 2019, American Society for Clinical Investigation.

Submitted: June 3, 2019 Accepted: September १९, 2019 Published: November 1, 2019.

Reference information: /CI Insight. 2019;4(21):e130811.

https://doi.org/10.1172/jici. insight.130811.

\section{Introduction}

Pancreatic ductal adenocarcinoma (PDAC) is projected to be the second leading cause of cancer-related mortality by 2030 (1) and presents as a highly heterogeneous disease with complex genetic and molecular diversity. Although the majority of PDACs share near-ubiquitous mutations of the KRAS oncogene and the frequent inactivation of TP53, SMAD4, and CDKN2A tumor suppressors, additional somatic mutations occur at low individual prevalence, suggesting diverse nongenetic mechanisms underlying PDAC progression (2). Recent large-scale transcriptomic analyses classified human PDAC into several molecular subtypes with distinctive histological and clinical characteristics (3-6). However, the molecular subtypes are not consistently associated with any somatic mutations or other genetically altered pathways (6), suggesting that the biological phenotypes of these subsets are driven by subtype-specific molecular mechanisms other than genetic alterations. Besides the aberrantly differentiated endocrine exocrine/exocrine (ADEX/exocrine) and immunogenic subtypes, which are likely defined by signatures derived from non-neoplastic cells 
$(4,6)$, the molecular signatures of cancer cells largely fall into 2 categories: the squamous/quasimesenchymal/basal-like and the progenitor/classical subtypes. The squamous subtype reproducibly exhibits the worst prognosis compared with the other subtypes $(3,5,6)$. Although the essential role of $K R A S$ oncogene in tumor initiation and maintenance has been well appreciated $(7,8)$, it has been recently demonstrated that $K R A S$ is dispensable for the survival of squamous subtype tumors $(5,9)$, suggesting that additional oncogenic drivers define and contribute to the malignancy of this subtype. Identifying the oncogenic pathways that drive the squamous subtype tumors will likely reveal subtype-specific vulnerabilities to treat these highly malignant tumors.

Yes-associated protein 1 (YAP1) is a transcriptional coactivator and plays critical roles in controlling normal tissue growth as well as tumor development (10). Its activity is kept in check by the upstream Hippo pathway, composed of the MST1/2-LATS1/2 kinase cascade, which phosphorylates YAP1 at multiple serine residues and sequesters YAP1 in cytoplasm for degradation (11). In vivo studies using genetically engineered mouse (GEM) models have shown that pancreas-specific Yap1 depletion abolished PDAC development driven by oncogenic Kras $(12,13)$, suggesting that YAP1 is essential for tumor initiation. However, the function of YAP1 in tumor maintenance in advanced human PDAC has not been firmly established. Notably, although we recently demonstrated that amplification of Yap1 gene is capable of bypassing KRAS dependency to maintain tumor growth in a PDAC mouse model (14), the genetic alterations in YAP1 and core components of its upstream Hippo signaling pathway are very rare in human PDAC, pointing to a critical need to identify the nature of YAP expression and regulation as well as its association with clinical outcomes in human PDAC.

In this study, we found that the YAP1 activation signature is highly enriched and preferentially required for the progression and maintenance of the squamous subtype of PDAC. Gene expression profiling further uncovered a strong positive correlation of the noncanonical WNT pathway with the YAP1 activation signature; WNT5A, a prototypical noncanonical WNT ligand, is significantly upregulated in YAP1-activated tumors and is required for YAP1 activation and tumorigenic activity in the squamous PDAC subtype. Moreover, we demonstrated that WNT5A enables the bypass of KRAS dependency to promote cell proliferation in vitro and drive tumor relapse in vivo in a YAP1-dependent manner. Our study delineated a critical role of the WNT5A/YAP1 axis in this deadliest form of human PDAC and identified context-specific vulnerabilities that may be exploited therapeutically.

\section{Results}

YAP1 plays a critical role in PDAC progression. We first evaluated the expression and role of YAP1 in human PDAC by using tissue microarray (TMA) analysis in a cohort of 92 human PDAC samples. As shown in Figure 1, 43 of 92 PDACs (47\%) exhibited high YAP1 protein expression in tumor epithelium compared with the surrounding tissue; the median overall survival for the YAP1-low group was 38.3 months compared with 25.3 months for the YAP1-high group $(P=0.02)$ (Figure 1, A and B). Such association between elevated YAP1 protein and poor survival is similar to a recent report (15) and was further validated in an independent cohort of 83 patients with PDAC $(P=0.0475)$ (Figure $1 C)$, suggesting that YAP1 may promote adverse biological outcomes in PDAC. We further characterized the in vivo function of YAP1 using GEM models. To faithfully recapitulate the PDAC initiation of human patients and investigate the requirement of YAP1 for PDAC when tumor initiation had started in the adult pancreas, we generated a tamoxifen-inducible YAP1-knockout mouse model of PDAC. Tamoxifen-induced acinar-specific activation of Cre recombinase in adult pancreas of the Mist1-Cre ${ }^{E R T 2} L S L-K r a s^{G 12 D /+} L S L-T r p 53^{R 172 H /+}(\mathrm{MKP})$ model led to rapid PDAC development accompanied by induction of nuclear YAP1 expression in tumor cells (YAP-WT; Supplemental Figure 1A; supplemental material available online with this article; https://doi. org/10.1172/jci.insight.130811DS1). In contrast, acinar-specific deletion of Yap1 in the MKPY (YAP-KO) model completely blocked tumor development (Supplemental Figure 1, A-C). Although all MKP mice succumbed to PDAC with a median survival of 103 days $(n=16)$, the Yap1-null MKPY mice remained entirely free of any overt pathological lesion $(n=13)$ (Supplemental Figure 1D). This could be attributed to the effect of YAP1 on cell proliferation and survival, as evidenced by the loss of both Ki67 and Survivin (BIRC5) staining in YAP-KO pancreas (Supplemental Figure 1, E and F). The protective effect of Yap1 deletion on $\mathrm{Kras}^{G 12 D_{-}}$and Trp53 $3^{R 172 H /+}$-induced PDAC development was further supported with an independent tamoxifen-inducible Elastase-Cre ${ }^{E R T 2} L S L-K r a s^{G 12 D /+} L S L-T r p 53^{R 172 H /+}$ model (Supplemental Figure 1G). These results suggested that YAP1 plays a critical role in PDAC progression. 
A
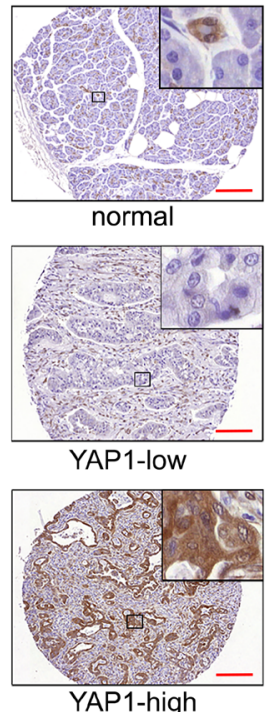

YAP1-high

B

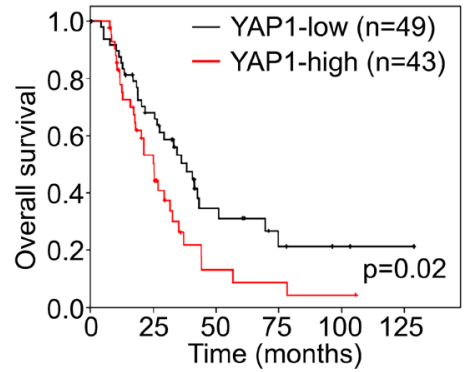

C

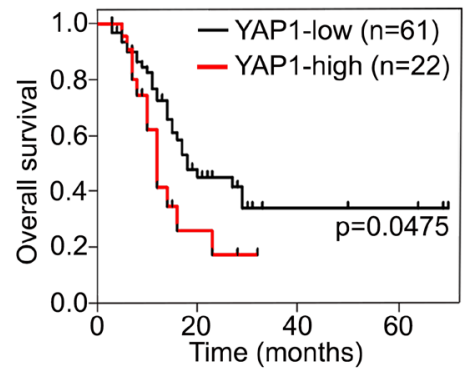

$\mathbf{F}$

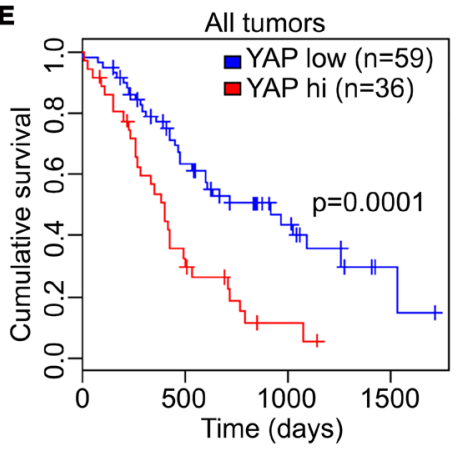

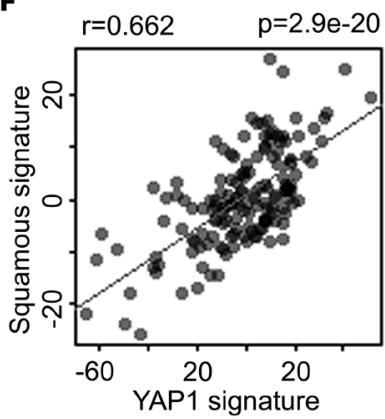
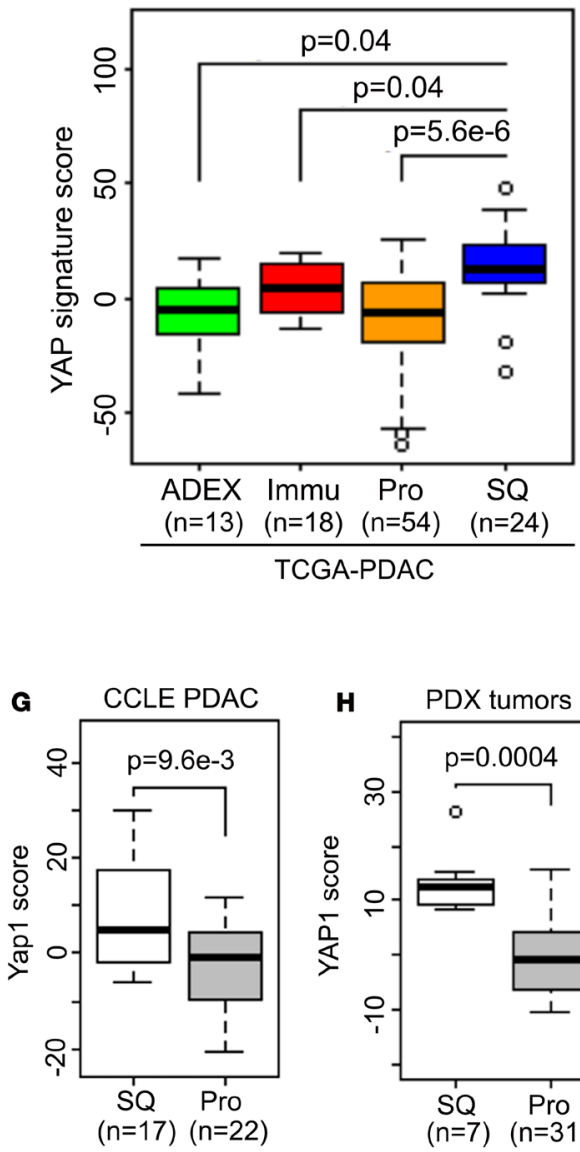

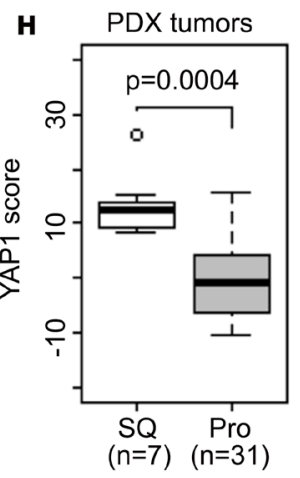

Figure 1. YAP1 is activated in the squamous subtype of human PDAC. (A) Representative images of YAP1 TMA showing tumor adjacent normal pancreatic tissue or tumor samples with low/high YAP1 level. Scale bar: $200 \mu \mathrm{m}$. (B and C) Kaplan-Meier curves for overall survival in patients with PDAC from MD Anderson (B) or Johns Hopkins University School of Medicine (C) stratified by YAP1 expression. (D) YAP1 signature score among human PDAC subtypes in The Cancer Genome Atlas (TCCA) data set. ADEX, aberrantly differentiated endocrine exocrine subtype; Immu, immunogenic subtype; Pro, progenitor subtype; SQ, squamous subtype. (E) Kaplan-Meier curves for overall survival in all patients with PDAC from TCGA data set stratified by YAP1 activation signature score. (F) Correlation between squamous subtype signature and YAP1 signature in PDAC TCGA data set. (G and H) YAP1 signature score in squamous or progenitor subtype human PDAC cell lines (G) or PDXs (H). The box plots depict the minimum and maximum values (whiskers), the upper and lower quartiles, and the median. The length of the box represents the interquartile range. Error bars for all panels indicate \pm SD. $P$ value for survival analysis was calculated with log-rank test.

YAP1 is activated in the squamous subtype of human PDAC. To further study the role of YAP1 in advanced human PDAC, we analyzed the expression profiles of the distinct molecular subtypes of human PDAC from TCGA collection (4) and found that tumors of the squamous subtype exhibited elevated expression of genes that are known to be associated with YAP1 activation (ref. 16, Figure 1D, and Supplemental Table 1). Consistent with the TMA analysis, YAP1 pathway activation was significantly correlated with poor survival in patients with PDAC (Figure 1E). Moreover, expression of the YAP1 activation signature was significantly correlated with that of the squamous subtype signature (Figure 1F), underscoring the tight association between YAP1 activation and squamous subtype tumors.

To further exclude the possibility that the YAP1 activation signature in squamous subtype PDAC is largely derived from tumor stroma, we analyzed the transcriptome data of human PDAC cell lines from the Cancer Cell Line Encyclopedia data set and of a collection of 47 PDAC patient-derived xenograft (PDX) models, after the expression reads from murine hosts were omitted. Consistent with the notion that the molecular signatures of ADEX or immunogenic subtypes are likely derived from nontumor cells (4), we failed to identify these signatures in both human PDAC cell lines and PDX models (Supplemental Figure 
2, A and B, and data not shown). Not surprisingly, the molecular signatures of human PDAC cell lines and PDX models clustered primarily under either the progenitor or squamous subtype (Supplemental Figure 2, A and B). In accordance with analysis of TCGA data, the YAP1 activation signature was consistently elevated in the squamous subtype cells (Figure 1, G and $\mathrm{H}$ ), underscoring that YAP1 is preferentially activated in the squamous PDAC subtype.

$Y A P 1$ is essential for the maintenance of the squamous subtype PDAC. To further investigate the requirement of YAP1 in the squamous subtype of PDAC, we conducted loss-of-function studies with shRNA in a panel of human PDAC cell lines and early-passage primary cell lines derived from human PDX tumors. Knockdown of YAP1 strongly suppressed the colony formation capacity of PDAC cell lines (PaTu8988T, SNU410, and PL45) and PDX cell lines (PATC148 and PATC153) belonging to the squamous subtype with a strong YAP1 activation signature (Figure 2, A and B, and Supplemental Figure 3, A and B). In contrast, the progenitor subtype cell lines, including PaTu8988S, HPAF-II, HPAC, PATC102, and PATC108 cells, were relatively insensitive to YAP1 depletion (Figure 2, A and B, and Supplemental Figure 3, A and B). Moreover, inducible knockdown of YAP1 in established tumors resulted in inhibition of proliferation, induction of apoptosis, and eventual regression of PaTu8988T tumors, whereas the growth of PaTu8988S tumors was not affected (Figure 2C and Supplemental Figure 3, C-E), indicating the role of YAP1 for tumor maintenance in the squamous PDAC subtype.

We further investigated whether YAP1 is able to endow progenitor subtype cells with the squamous phenotype. Expressing constitutive active YAP1 $1^{\mathrm{S} 127 \mathrm{~A}}$ mutant, which is resistant to cytoplasmic retention and degradation (11), in PaTu8988S cells, a progenitor subtype human PDAC cell line, induced a transcriptional signature that was highly similar to that of PaTu8988T cells, which were derived from the same patient as PaTu8988S cells (17) but were of the squamous subtype (Figure 2D and Supplemental Table 2). Of importance, ectopic expression of YAP1 $1^{\mathrm{S} 127 \mathrm{~A}}$ substantially enhanced the anchorage-independent growth, migration, and invasion capacity of PaTu8988S cells and an early-passage patient-derived cell line, Pa04C (Figure 2, $\mathrm{E}$ and F, and Supplemental Figure 3, F and G), suggesting active YAP1 may promote the tumorigenicity and metastatic spread of PDAC cells. Indeed, YAP1 ${ }^{\text {s127A }}$ expression diminished necrotic regions within the primary tumor core and enhanced the distal metastasis of Pa04C cells in an orthotopic xenograft model (Supplemental Figure 3, H-J). This was accompanied by induction of canonical YAP1 target genes, such as CYR61, CTGF, and $A X L$, in both cultured cells and xenograft tumors (Supplemental Figure 3, $\mathrm{K}$ and $\mathrm{L}$ ). Gene expression microarray and subsequent gene set enrichment analysis (GSEA) in these cells supported an established YAP1 signature (Supplemental Figure 3M), enrichment in pathways associated with tumor development and metastasis, and the underlying cellular processes responsible, such as cell proliferation, cell cycle progression, migration, motility, and epithelial-mesenchymal transition (Supplemental Tables 3 and 4). Of interest, cellular processes including cell cycle progression and signaling pathways significantly activated in Pa04C-YAP1 ${ }^{\text {S127A }}$, such as MYC, IL-6/STAT3, TGF- $\beta$, RhoA, E2F, and TNF, along with the Hippo signaling pathway, were part of all 4 gene signatures (GP2-5) associated with the squamous subtype of PDAC (ref. 3 and Supplemental Tables 5 and 6), underscoring the role of YAP1 activation in this most aggressive subtype.

We and others have previously shown that YAP1 activation enables the bypass of oncogene addiction in multiple cancer types, including PDAC (14, 18-20). Indeed, compared with PaTu8988S cells, PaTu8988T cells were more resistant to KRAS knockdown with shRNA (Supplemental Figure 4, A-C). Expression of YAP1 ${ }^{\text {S127A }}$ partially rescued the growth of PaTu8988S cells upon KRAS depletion (Supplemental Figure 4, B and C), indicating that YAP1 activation could enable the bypass of KRAS dependence in human PDAC cells. Accordingly, pathway analysis of human PDAC expression profiles in TCGA data set indicated that gene signatures induced upon KRAS knockdown (21) or suppressed by oncogenic KRAS expression (22) were significantly upregulated in squamous subtype tumors (Supplemental Figure 4D), suggesting relatively low KRAS activity in these tumors. By using an inducible Kras $^{G 12 D}$-driven PDAC GEM model, we recently obtained a collection of spontaneous relapse tumors following $\mathrm{Kras}^{G 12 D}$ extinction in advanced PDAC (inducible-Kras-negative tumors; iKras ${ }^{-}$tumors) (14). These tumors neither expressed oncogenic Kras nor exhibited strong activation of KRAS surrogates and are thus deemed KRAS-independent. Of interest, the molecular signature of the iKras ${ }^{-}$tumors (Supplemental Table 7), which is composed of genes that are highly expressed in iKras ${ }^{-}$tumors compared with those in $\mathrm{KKras}^{+}$ones (14), was also significantly enriched in the squamous subtype of human PDACs (Supplemental Figure 4, E and F), further supporting the notion that squamous subtype tumors are relatively KRAS-independent. Consistent with YAP1 activation in the human squamous subtype of PDAC, 
A

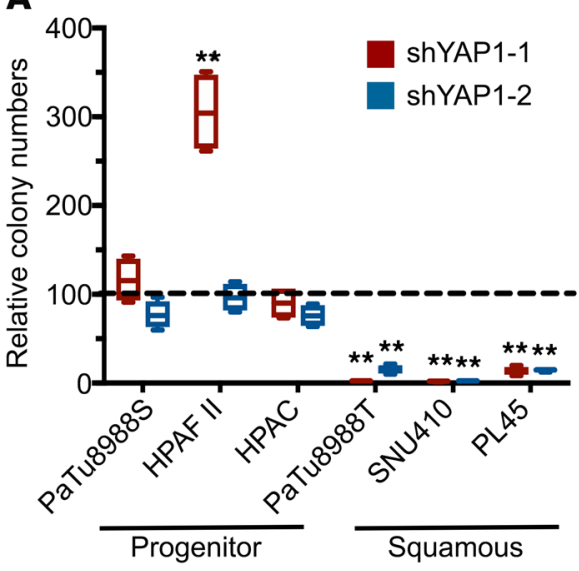

B

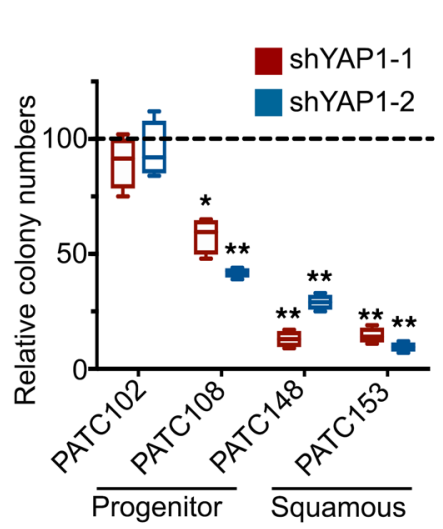

C

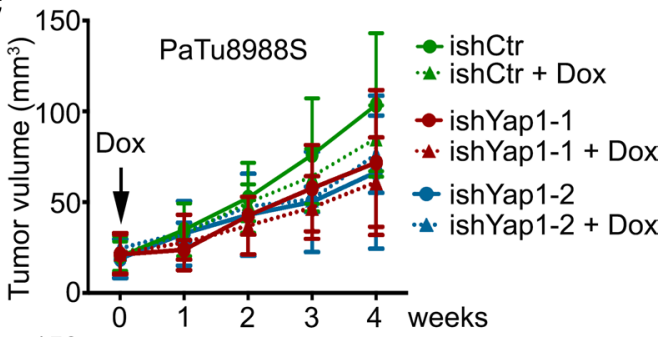

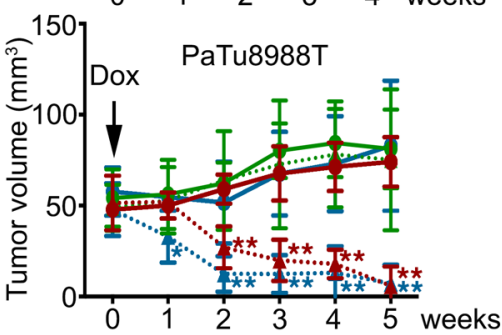

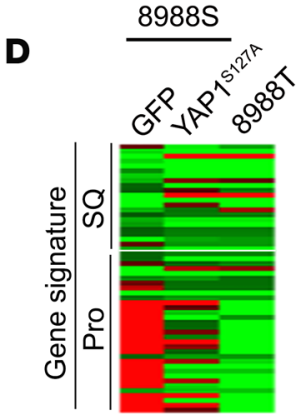

E

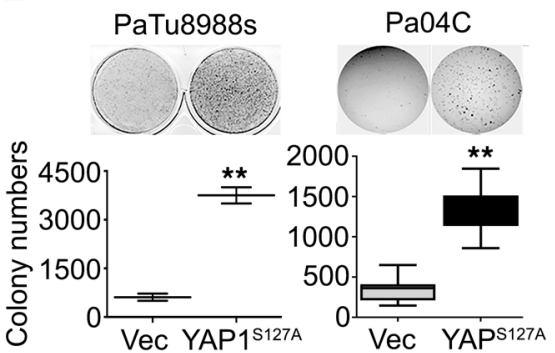

$\mathbf{F}$

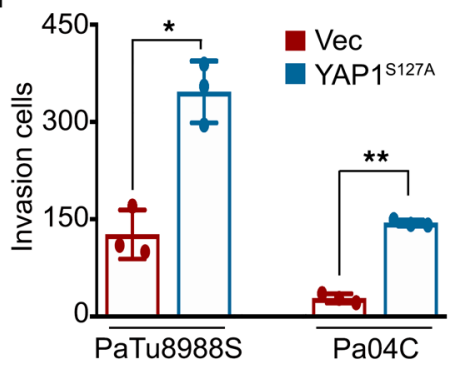

G

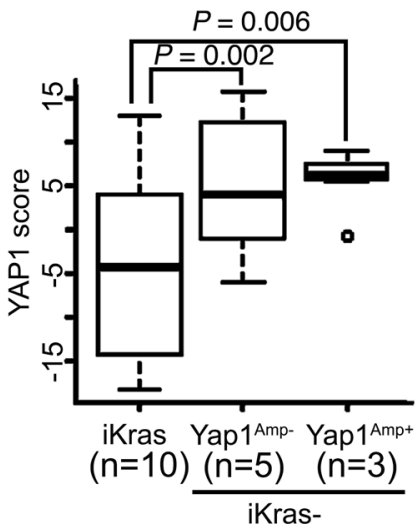

H

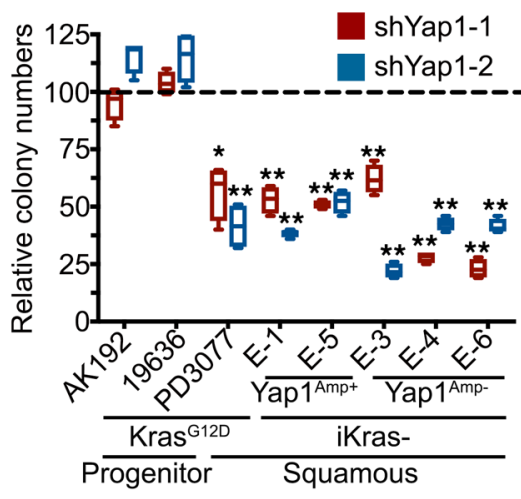

Figure 2. YAP1 is essential for the maintenance of squamous subtype of PDACs. (A and B) Colony formation assay in human PDAC cell lines (A) or PDX cells (B) infected with YAP1 shRNAs or nontargeting shRNA. Relative colony numbers upon normalization to the nontargeting shRNA group are shown (n = 3). (C) PaTu8988S and PaTu8988T cells engineered with inducible shRNA targeting YAP1 or nontargeting shRNA were subcutaneously injected into nude mice. Animals were treated with doxycycline once tumor size reached $20-50 \mathrm{~mm}^{3}$. Tumor volumes were measured on the indicated dates after injection $(n=5)$. (D) Heatmap shows the expression level of squamous and progenitor subtype signature genes measured with NanoString in PaTu8988S cells expressing GFP or YAP1 $1^{5127 A}$ and PaTu8988T cells. (E and F) Ectopic expression of YAP1 ${ }^{5127 A}$ in PaTu8988S and Pa04C cells promotes anchorage-independent growth in soft agar (quantification from triplicates shown) (E) and cell invasion in a Boyden chamber assay (quantification of triplicates is shown) (F). (C) YAP1 signature score among mouse PDAC tumors with indicated genotypes. (H) Colony formation assay in mouse PDAC cells infected with YAP1 shRNAs or nontargeting shRNA. Relative colony numbers upon normalization to the nontargeting shRNA group is shown $(n=3)$. The box plots depict the minimum and maximum values (whiskers), the upper and lower quartiles, and the median. The length of the box represents the interquartile range. Error bars from all panels indicate $\pm \mathrm{SD}$. ${ }^{*} P<0.05 ;{ }^{* *} P<0.01$.

the YAP1 signature was significantly enriched in mouse iKras ${ }^{-}$tumors (Figure 2G). Although YAP1 knockdown exhibited minimal effect on progenitor subtype Kras-driven mouse tumor lines (AK192 and 19636), YAP1 depletion significantly suppressed the proliferation and colony formation capability of squamous subtype cells (PD3077; ref. 23) and iKras tumor cells (Figure 2H and Supplemental Figure $4 \mathrm{G})$, underscoring YAP1 dependency in the squamous subtype of tumors. 
WNT5A overexpression contributes to YAP1 activation in PDAC. To identify mechanisms for YAP1 activation, we first performed genomic analysis of primary human tumors from TCGA data set, showing that no frequent copy number changes or mutations of the YAP1 locus were revealed (Supplemental Figure 5A). Similarly, although Yap1 is amplified in a subset of mouse iKras ${ }^{-}$tumors (14), most iKras ${ }^{-}$tumors harbor no genomic alteration of Yap1 despite enrichment of the YAP1 signature (Figure 2G and Supplemental Figure 5B). In addition, there is no obvious difference in YAP1 transcription level between squamous and progenitor subtypes of PDAC cell lines (Supplemental Figure 5C), indicating posttranscriptional mechanisms for YAP1 activation in PDAC. Indeed, YAP1 activation as manifested by its nuclear translocation (11) was evident in YAP1-dependent human PDAC cells, including PaTu8988T, SNU410, and PL45 cells and $\mathrm{KKras}^{-}$mouse tumors or the derived cell lines without Yap1 amplification ( KKras $^{-}$Yap $^{\text {Amp- }}$ ) (Figure 3, A and B, and Supplemental Figure 5D). Conversely, YAP1 was localized in both the cytoplasm and nuclei in PDAC cells with weak YAP1 signature, such as PaTu8988S, HPAF-II, and HPAC cells, as well as progenitor subtype $K r a s^{G 12 D}$-driven mouse tumors and $i \mathrm{Kras}^{-}$tumors with Yap1 amplification ( KKas $^{-}$Yap $^{\text {Amp }^{A}}$ ) (Figure 3, A and B). Accordingly, YAP1-activated human and mouse PDAC cells exhibited reduced phosphorylation at S127 (Supplemental Figure 5, E and F), suggesting that YAP1 activation is largely mediated by the upstream Hippo pathway at the posttranslational level.

We further performed GSEA analysis to compare the differentially regulated pathways in $i$ Kras Yap$1^{\text {Amp- }}$ versus $i K r a S^{-}$Yap $^{\text {Amp }+}$ cells. Of interest, one of the pathways significantly elevated in $i K r a S^{-}$Yap $^{\text {Amp- }}$ cells was the noncanonical WNT pathway (ref. 24, Figure 3C, and Supplemental Table 8), which was recently shown to suppress Hippo signaling and activate YAP1 in adipocytes (25). Of importance, a significant correlation between the noncanonical WNT signature and YAP1 activation signature was observed in TCGA data set (Figure 3D), suggesting control of YAP1 activation by the noncanonical WNT pathway in PDAC. A survey of WNT ligands identified WNT5A, the prototypic noncanonical WNT ligand (26), to be exclusively overexpressed in $i \mathrm{Kras}^{-} \mathrm{Yap}^{\text {Amp- }}$ tumor cells at both mRNA and protein levels (Figure 3E and Supplemental Figure 5G). Moreover, WNT5A expression was also significantly elevated in the squamous subtype compared with its expression in progenitor subtype tumors and was significantly correlated with the YAP1 signature in TCGA data set (Figure 3, F and G). Deletion of Wnt5a with CRISPR in 2 independent $i$ Kras Yap $1^{\text {Amp- }}$ tumor cell lines led to the induction of YAP1 phosphorylation at S127, the increase in cytoplasmic retention of YAP1 protein, as well as the downregulation of YAP1 downstream target genes (Figure 3, H-J). In addition, ectopic expression of Wnt5a in Kras ${ }^{G 12 D}$-driven tumor cells resulted in a decrease in YAP1 phosphorylation (Figure 3K), further supporting the notion that WNT5A expression drives YAP1 activation in mouse PDAC cells. We validated these findings in human PDAC cell lines, in which WNT5A expression was found to be highly elevated in the YAP1-dependent PaTu8988T cells in contrast to progenitor subtype PaTu8988S cells (Figure 3E). Ectopic expression of WNT5A in PaTu8988S cells reduced YAP1 phosphorylation and enhanced YAP1 nuclear localization (Figure 3, I and K). In contrast, depletion of WNT5A in PaTu8988T cells with shRNA resulted in elevated YAP1 phosphorylation with concordant induction of LATS1/2 phosphorylation (Figure 3L), supporting the notion that WNT5A activates YAP1 through the suppression of Hippo signaling. Taken together, our data suggest that WNT5A overexpression can lead to YAP1 activation in PDAC cells.

WNT5A overexpression enables tumor maintenance and bypass of KRAS dependence. At the functional level, Wnt5a deletion in $i \mathrm{Kras}^{-}$Yap $^{\text {Amp- }}$ tumor cells with CRISPR significantly inhibited colony formation (Figure 4, A and B). In agreement with the genetic ablation, treatment with WNT5A antagonist BOX-5 specifically induced YAP1 phosphorylation and abolished the colony formation ability of $i$ Kras Yap $1^{\text {Amp }}$ - tumor cells, but not the Yap $1^{\text {Amp }}$ cells (Figure 4, C-E), indicating the requirement of WNT5A for YAP1 activation and induced tumorigenic activity. Indeed, $W n t 5 a$ deletion also significantly inhibited xenograft tumor growth in vivo, which was rescued by reconstituted WNT5A expression (Figure 4, F and G). Of importance,

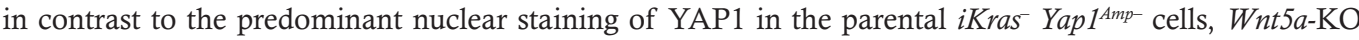
tumors exhibited a significant amount of cytoplasmic YAP1, whereas WNT5A reconstitution restored YAP1 nuclear accumulation without affecting total YAP1 expression level (Figure 4, H and I, and Supplemental Figure 6A), suggesting that the diminished tumor growth was due to decreased YAP1 activity. Consistent with the role of YAP1 in driving squamous subtype, expression of squamous subtype genes (Cav1 and Pappa) was suppressed in $W n t 5 a$-KO tumors whereas the expression of progenitor subtype genes (Tff1 and Muc13) was induced, which was partially reversed upon WNT5A reconstitution (Supplemental Figure $6 \mathrm{~B})$. In addition, expression of constitutively active YAP $1^{\text {S127A }}$ largely rescued the inhibitory effect of $W n t 5 a$ 


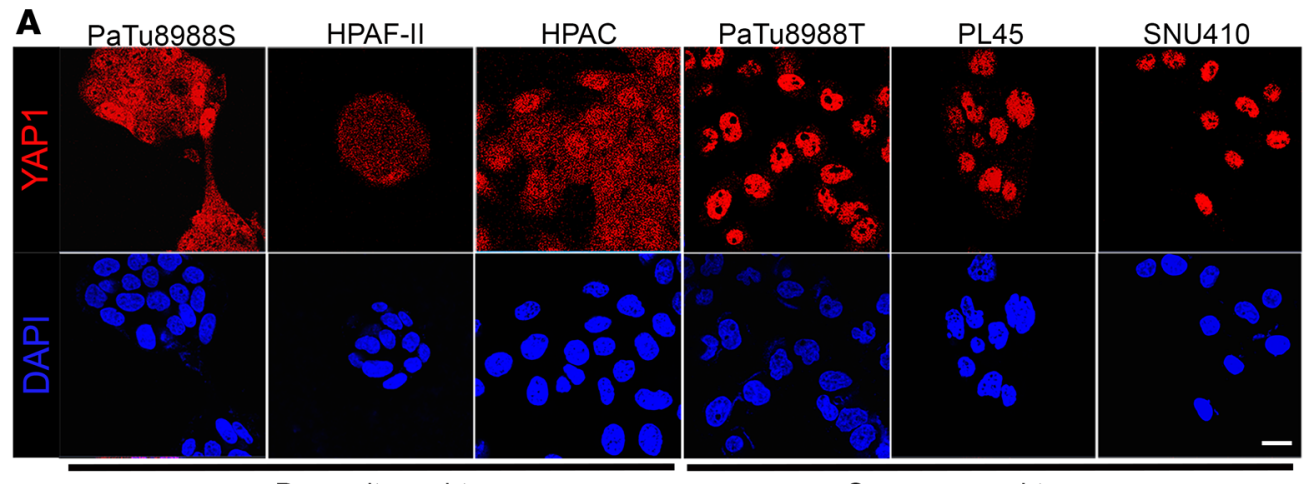

Progenitor subtype

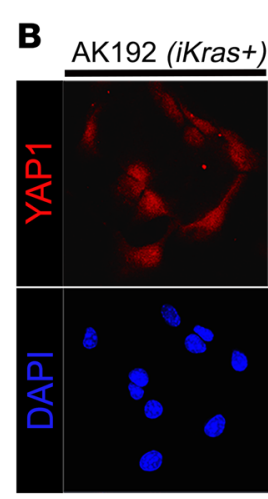

iKras- Yap $1^{\text {Amp+ }}$

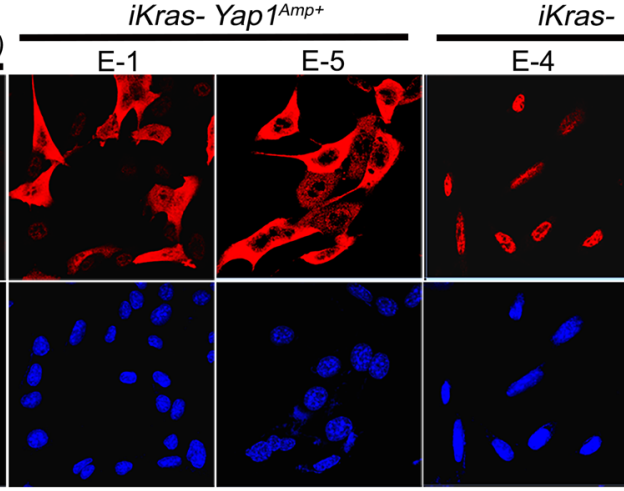

Squamous subtype

Kras- Yap1 $1^{\text {Amp- }}$

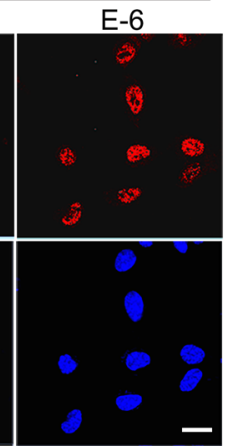

C iKras-
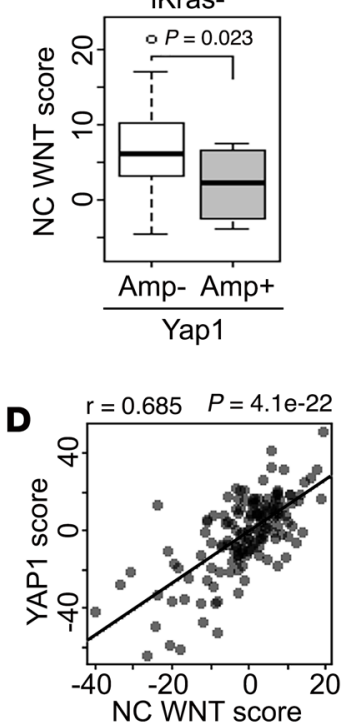

E

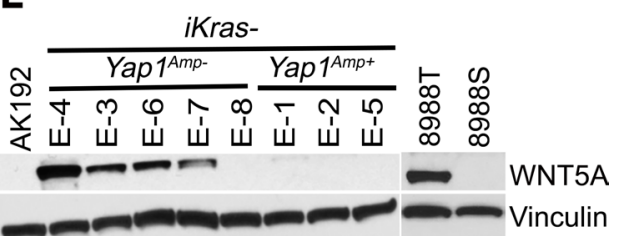

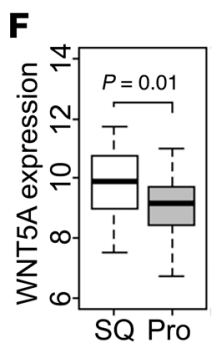

$(n=49)(n=50)$
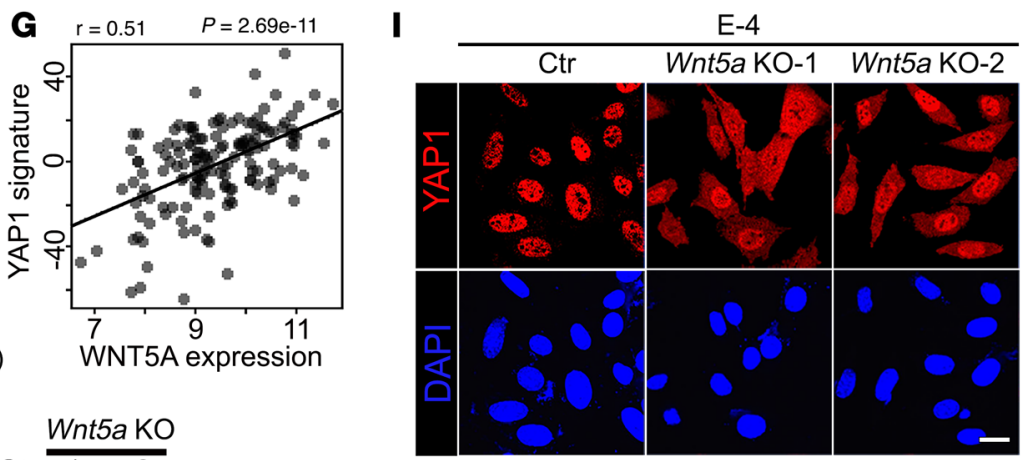

PaTu8988S

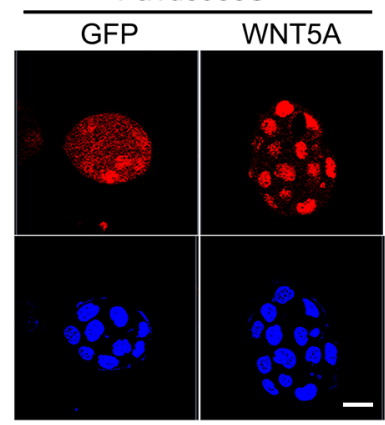

H $\operatorname{Ctr} \frac{W n t 5 a \mathrm{KO}}{12} \mathrm{Ctr} \frac{W n t 5 a \mathrm{KO}}{12}$

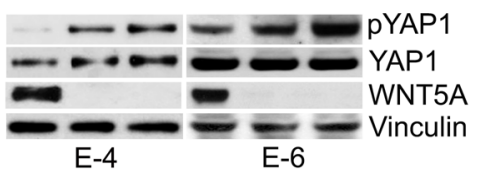

J

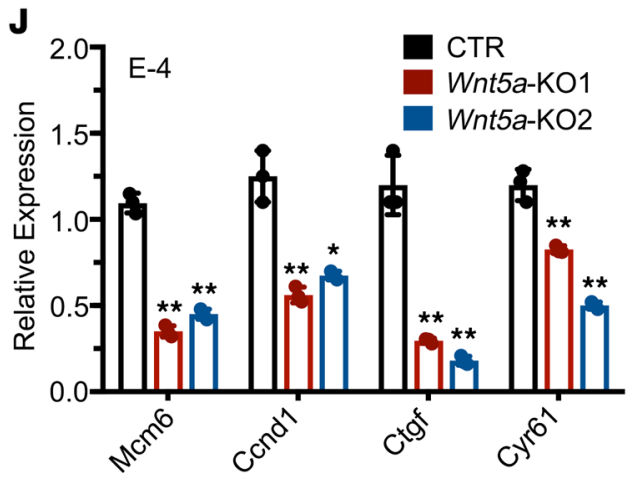

AK192 PaTu8988S

K
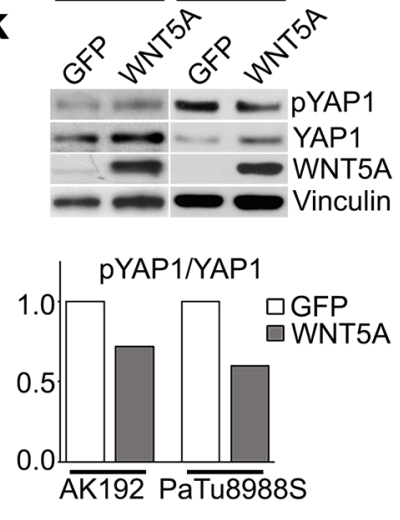

$\mathbf{L}$

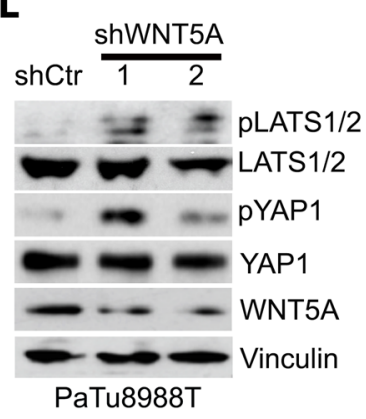


Figure 3. YAP1 activation in PDAC is mediated by WNT5A overexpression. (A and B) Human pancreatic cancer cell lines of progenitor or squamous subtype (A) or mouse PDAC cells of indicated genotypes (B) were subjected to immunofluorescence staining with anti-YAP1 (red) and DAPI (blue). (C) Noncanonical (NC) WNT pathway enrichment score in iKras- mouse PDAC tumors without (Amp-) or with (Amp+) Yap1 amplification. (D) Correlation between NC WNT signature and YAP1 signature in PDAC TCGA data set. (E) Western blots for WNT5A in mouse PDAC cells of indicated genotypes and human PDAC cell lines. (F) WNT5A expression in squamous or progenitor subtype human PDACs in TCGA data set. (C) Correlation between WNT5A expression and YAP1 signature in PDAC TCGA data set. (H) Western blot analysis for WNT5A, YAP1, and phospho-YAP1 (S127) in 2 independent $i K r a s-Y a p{ }^{\text {Amp- }}$ cells with CRISPR-mediated Wnt5a deletion. Two independent Wnt5a-knockout (KO) clones were included, and a clone without Wnt5a deletion was used as a control (Ctr). Vinculin blot for E-4 was run in parallel with the rest of the blots for E-4 contemporaneously. (I) E-4 (iKras-Yap1 ${ }^{\text {Amp-) }}$ Wnt5a-KO cells and PaTu8988S cells expressing GFP or WNT5A were subjected to immunofluorescence staining with anti-YAP1 (red) and DAPI (blue). (J) Relative mRNA levels of YAP1 downstream targets in E-4 (iKras ${ }^{-}$Yap$1^{\text {Amp-) }}$ Wnt5a-KO cells. $P$ value was corrected with Dunnett's method. (K) Western blot analysis for WNT5A, YAP1, and phospho-YAP1 (S127) in mouse iKras PDAC cells (AK192) or human PaTu8988S cells expressing GFP or WNT5A (top). The quantification of phospho-YAP1/total YAP1 signals is shown. (L) Western blot analysis for WNT5A, LATS1/2, phospho-LATS1/2, YAP1, and phospho-YAP1 (S127) in PaTu8988T cells infected with WNT5A shRNAs or nontargeting shRNA (shCtr). The box plots depict the minimum and maximum values (whiskers), the upper and lower quartiles, and the median. The length of the box represents the interquartile range. Error bars from all panels indicate $\pm S D$. ${ }^{*} P<0.05$; ${ }^{* *} P<0.01$. Scale bar: $20 \mu \mathrm{m}$.

deletion on tumor growth (Figure 4G), thus suggesting that WNT5A overexpression in mouse PDAC cells promotes tumor growth by activating YAP1. In agreement with this notion, depletion of WNT5A in human PDAC cell line PaTu8988T significantly inhibited cell colony formation ability (Figure 4J).

Because YAP1 activation can maintain tumor growth upon genetic extinction of KRAS oncogene in PDAC $(14,20)$, we next investigated whether WNT5A overexpression can also serve to bypass KRAS dependency. Indeed, ectopic expression of WNT5A in Kras ${ }^{G 12 D}$-driven iKras tumor cells and KRAS-dependent PaTu8988S cells partially restored the colony formation upon KRAS depletion (Figure 5A and Supplemental Figure 6, C and D). In addition, forced WNT5A expression in Kras ${ }^{12 D}$-driven iKras tumor spheres was able to maintain cell viability upon extinction of $\mathrm{Kras}^{G 12 D}$ by doxycycline withdrawal, whereas most control tumor cells expressing GFP underwent apoptosis (Figure 5, B-D). Of importance, the survival effect of WNT5A upon Kras ${ }^{G 12 D}$ extinction was largely abolished upon YAP1 knockdown (Figure 5, B-D), indicating that YAP1 is required for WNT5A-induced bypass of KRAS dependence. Indeed, similar to the effect of YAP1 ${ }^{\text {S127A }}$, ectopic WNT5A expression in iKras tumor cells showed KRAS-independent tumor growth when injected orthotopically into nude mice, whereas GFP-expressing iKras tumor cells failed to maintain tumor growth in the absence of doxycycline (Figure 6, A-C). Of importance, WNT5A-induced KRAS-independent tumor growth was abolished in Yap1-deleted cells (Supplemental Figure 6, E and F), underlining the requirement of YAP1 for WNT5A-mediated bypass of KRAS dependence. Notably, all WNT5A-driven tumors showed lower MAPK activity and strong

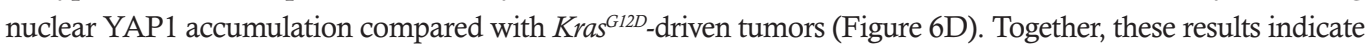
that WNT5A overexpression can activate YAP1 and substitute for oncogenic Kras-driven tumor maintenance.

WNT5A/YAP1 axis functions in primary human PDAC. We further validated the WNT5A/YAP1 axis in primary human PDAC, showing that WNT5A expression was elevated in squamous subtype PDX tumors (Figure 7A). Accordingly, WNT5A protein was highly expressed, whereas YAP1 phosphorylation was relatively low in squamous subtype PATC148 and PATC153 cells, while 2 cell lines derived from progenitor subtype tumors, PATC102 and PATC108, exhibited elevated YAP1 phosphorylation along with absence of WNT5A expression (Figure 7B). This is in accordance with the elevated expression of YAP1 target gene CYR61 in squamous subtype PDXs (Figure 7B).

ShRNA-mediated depletion of WNT5A in PDX-derived PATC148 and PATC153 cells caused an increase in YAP1 phosphorylation, suppression of colony formation ability, and diminished tumor growth in vivo (Figure 7, C-F), supporting the role of WNT5A for tumorigenic activity. In contrast, WNT5A shRNA had minimal effect on in vivo tumor growth of PATC108 cells (Figure 7F). Consistent with the role of WNT5A in bypass of KRAS dependence, knockdown of KRAS elicited less inhibition on the growth of high WNT5A-expressing PATC148 (KRAS $\left.{ }^{\text {G12D }}\right)$ cells compared with low WNT5A-expressing PATC102 $\left(K R A S^{G 12 D}\right)$ and PATC108 $\left(K R A S^{G 12 D}\right)$ cells, with KRAS-WT PATC153 cells being resistant to KRAS knockdown (Figure 7, G and H). Together, our data indicate that WNT5A overexpression in squamous subtype PDACs contributes to YAP1 activation and tumor growth.

\section{Discussion}

In this study, we found that deletion of Yap 1 in adult pancreas completely blocked KRAS-induced PDAC development (Supplemental Figure 1, B-D). Immunohistochemical staining on these tumor tissues showed marked decrease in the cell proliferation index, as measured by Ki67 staining (Supplemental Figure 1F) and low Survivin (BIRC5) expression (Supplemental Figure 1E). Because Survivin expression overlapped 

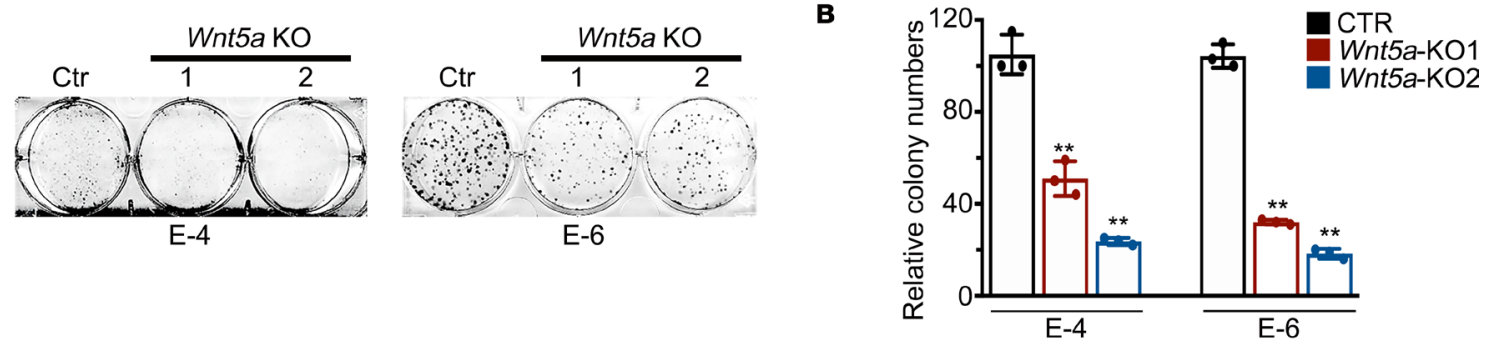

C

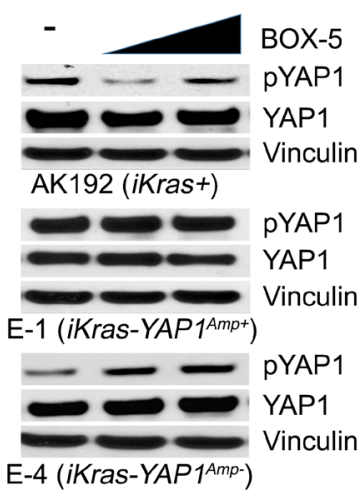

D

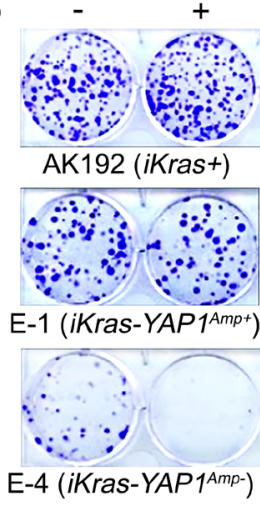

$\mathbf{F}$

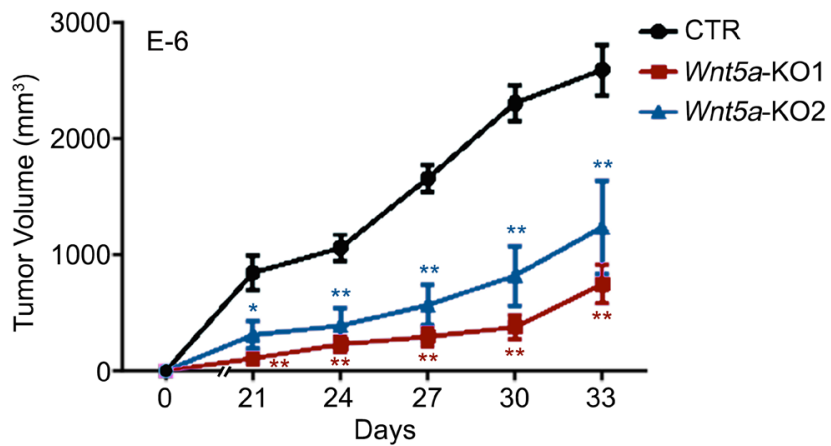

H

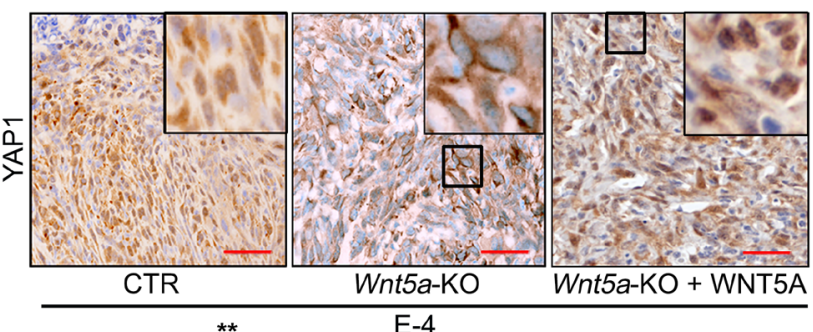

I

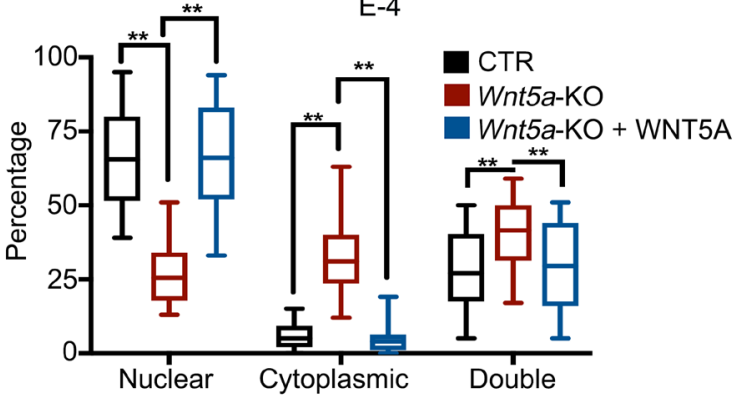

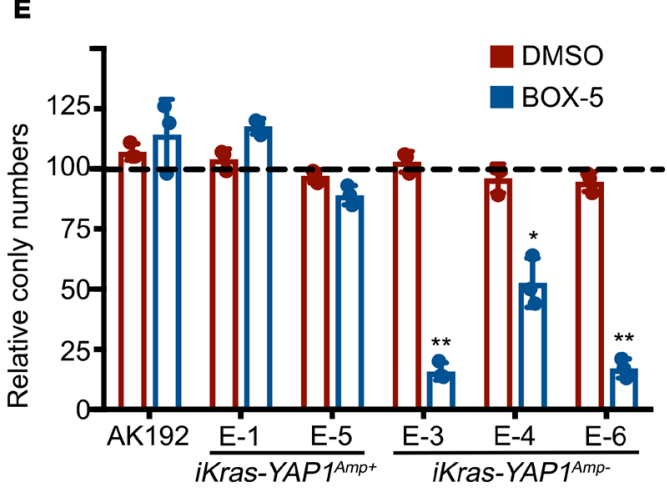
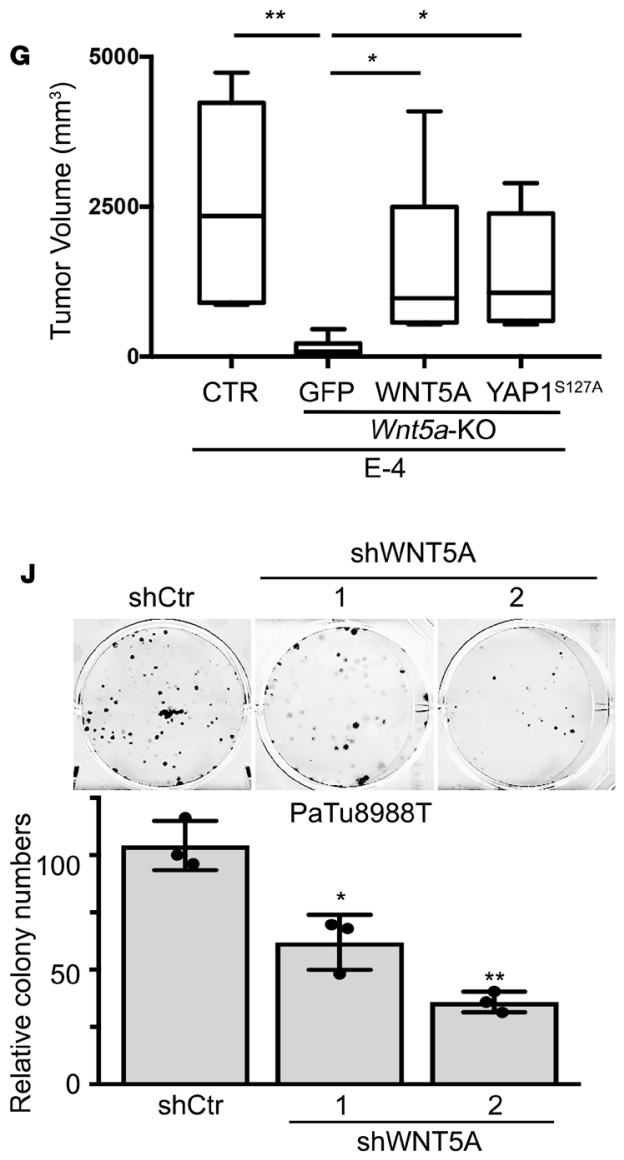
Figure 4. WNT5A overexpression is required for tumorigenic activity. (A) Representative images of the colony formation assay for ( $\left.i K \mathrm{Kras}^{-} \mathrm{Yap}^{\mathrm{Amp}-}\right)$ Wnt5a$\mathrm{KO}$ cells. Clones without Wnt5a deletion were used as a control. (B) Quantification from triplicates is shown and is presented as relative colony numbers upon normalization to the shCtr group. (C) Western blot analysis for YAP1 and phospho-YAP1 in mouse PDAC cells treated with DMSO, 50 or 100 $\mu$ M BOX-5. (D) Representative images of the colony formation assay in mouse PDAC cells of indicated genotypes treated with vehicle (DMSO) or BOX-5 (100 $\mu$ M). (E) Quantification from triplicates is shown and is presented as relative colony numbers upon normalization to DMSO group. (F) Two independent clones of E-6 (iKras Yap ${ }^{A m p-}$ ) Wnt5a-KO cells and control cells without Wnt5a deletion (CTR) were subcutaneously injected into nude mice. Tumor volumes were measured on the indicated dates after injection. Results are presented as the means \pm SD $(n=5)$. (G) E-4 (iKras Yap $\left.^{\text {Amp }}{ }^{-}\right)$Wnt5a-KO cells were infected with GFP, WNT5A, or $Y A 1^{5127 A}$ and were subcutaneously injected into nude mice. Cells without $W n t 5 a$ deletion were used as a control. Tumor volumes were measured 30 days after injection. Results are presented as means \pm SD $(n=5)$. (H) Subcutaneous xenograft tumors from G were stained for YAP1. Scale bar: $100 \mu \mathrm{m}$. (I) Percentage of cells with nuclear/cytoplasmic/double staining of YAP1. Error bars represent SD ( $n=10$ fields, 250 cells/field). $P$ value was corrected with Dunnett's method. (J) Representative images of the colony formation assay for PaTu8988T cells infected with WNT5A shRNAs or nontargeting shRNA (shCtr) (top). Quantification from triplicates is shown. The box plots depict the minimum and maximum values (whiskers), the upper and lower quartiles, and the median. The length of the box represents the interquartile range. Error bars from all panels indicate $\pm \mathrm{SD}$. ${ }^{*} P<0.05 ;{ }^{*} P<0.01$.

with YAP1 expression in Yap1-WT PDAC but was completely lost in Yap1-KO pancreas, it is likely regulated directly by YAP1 at the transcription level, as shown in esophageal squamous cell carcinoma (27). Survivin is a known antiapoptotic protein that is expressed only in tumor cells (28) and primarily during the $\mathrm{G}_{2}$ mitotic phase of the cell cycle (29). The mostly nuclear expression of Survivin that is observed in YAPWT tumor sections (Supplemental Figure 1E) supports its predominant role in regulating the cell cycle. Accordingly, higher Survivin expression was also in agreement with predominant gene signatures associated with cell cycle progression in Pa04C-YAP1 ${ }^{\text {S127A }}$ cells (Supplemental Table 4) and a higher percentage of Pa04C-YAP1 ${ }^{\text {S127A }}$ cells in the $G_{2} / M$ phase by cell cycle analysis (data not shown).

We provided evidence that YAP1 is highly activated in squamous subtype PDACs and is required for their tumorigenic function. Despite the emerging role of $Y A P 1$ as a major oncogene in multiple cancer types, genetic alterations of the YAP1 gene or its upstream Hippo pathway are relatively uncommon (30, 31). YAP1 amplification or mutations in NF2, an upstream negative regulator of YAP1 activity, has been reported in about $1 \%$ of human PDACs $(3,32)$. Therefore, the activation of YAP1 in advanced human PDAC is likely due to nongenetic factors regulating inhibitory upstream Hippo kinases. Our data showing constitutive nuclear localization of YAP1 protein in YAP1-dependent tumor cells indicates that suppression of Hippo signaling is the major mechanism for YAP1 activation in PDAC. It was shown that TP63 ( $\triangle \mathrm{Np63}$ ) drives the squamous subtype of PDAC (33). Of interest, TP63 has been shown to activate YAP1 in head and neck squamous cell carcinoma through the suppression of Hippo signaling (34). Whether TP63 also functions through YAP1 activation in the squamous subtype of PDAC remains to be investigated. More recently, GLI2 transcriptional factor was demonstrated to drive the switch between progenitor and squamous subtypes in PDAC (35). Importantly, GLI2 was identified as a direct downstream target of YAP1 in medulloblastoma (36). It will be interesting to further investigate whether YAP1 also functions upstream of GLI2 in squamous subtype PDAC.

Here we provide evidence that WNT5A overexpression leads to YAP1 activation and bypass of KRAS dependency in KRAS-independent mouse PDAC cells and a subset of human squamous subtype PDACs. WNT5A is a prototypic noncanonical WNT ligand (26) and has been implicated in the pathogenesis of PDAC $(37,38)$. It was recently shown that the WNT5A-mediated noncanonical WNT pathway suppresses Hippo signaling and activates YAP1 through G protein-dependent activation of Rho GTPases (25). WNT5A can also engage multiple additional downstream signaling pathways, including SRC and PKC, which have been shown to activate YAP1 directly through phosphorylation or indirectly through regulation of Rho GTPases and LATS activity (39-44). Of interest, it was recently reported that noncanonical WNT- and frizzled class receptor 8-mediated (FZD8-mediated) calcium signaling counteracts the tumorigenic activity of oncogenic KRAS (45). In contrast, FZD1 was shown to be important for WNT5A-mediated YAP1 activation (25). It is possible that the engagement of specific receptors by WNT5A determines its signaling and biological output in PDAC. Whether any one or all of these mechanisms are responsible for WNT5A-mediated YAP1 activation in PDAC remains to be further studied. Furthermore, additional noncanonical WNT ligands are also likely involved in YAP1 activation in PDAC. For instance, WNT7B expression is also elevated in the squamous subtype of PDAC and correlated with the YAP1 activation signature (data not shown). It is worthwhile to determine whether the additional noncanonical WNT ligands also contribute to YAP1 activation in PDAC.

Although our data indicate that WNT5A overexpression in tumor cells functions in a cell-autonomous manner to activate YAP1 oncoprotein, tumor cells may also activate WNT5A signaling through paracrine mechanisms. Notably, WNT5A has been shown to be highly expressed in PDAC stroma fibroblasts (46, 
A

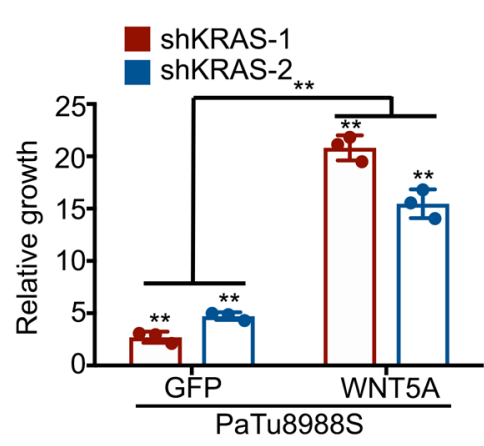

B

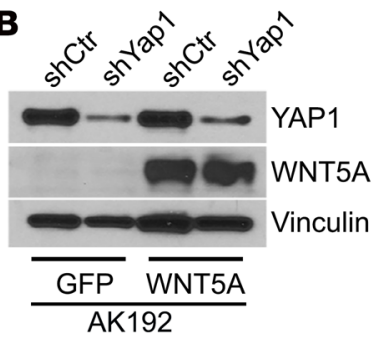

C

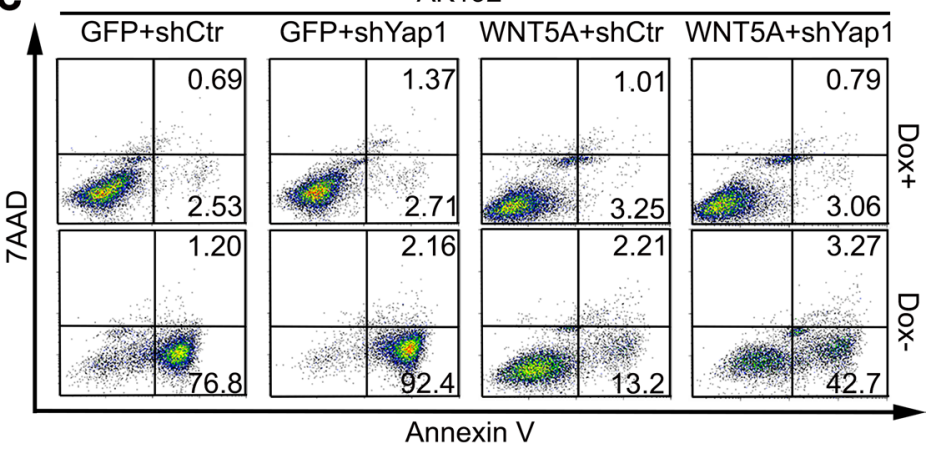

D

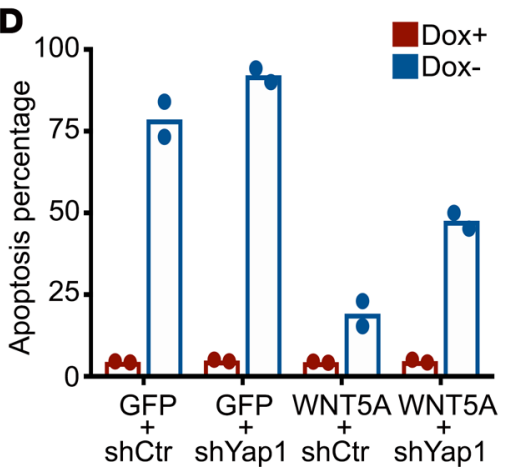

Figure 5. WNT5A overexpression leads to the bypass of KRAS dependency. (A) Cell growth assay for PaTu8988S-GFP or -WNT5A cells infected with KRAS shRNAs or nontargeting control shRNA. Quantification from triplicates is presented as relative cell growth upon normalization to control group. Error bars indicate \pm SD of triplicates; ${ }^{* *} P<0.01$. (B) Western blot analysis for WNT5A and YAP1 in AK192 (iKras ${ }^{+}$) cells expressing GFP or WNT5A upon knockdown of YAP1 with shRNA. (C) Mouse AK192-GFP or AK192-WNT5A cells infected with nontargeting shRNA (shCtr) or YAP1 shRNA (shYap1) were grown as 3D tumor spheres in the presence or absence of doxycycline for 4 days. Cellular apoptosis was measured with annexin $V$ staining. Representative images of 2 independent experiments show the FACS analysis of annexin $V$ and 7-AAD staining. Numbers represent the percentage of early apoptosis (annexin $\mathrm{V}^{+}$7-AAD) and late apoptosis (annexin $\mathrm{V}^{+}$7-AAD+) populations. (D) Quantification of total apoptotic cell from C ( 2 independent experiments). Doxycycline withdrawal leads to dramatic apoptosis of AK192-GFP tumor sphere. Such apoptosis induced by doxycycline withdrawal was significantly inhibited in WNT5A-expressing cells, which was partially reversed upon YAP1 knockdown. The box plots depict the minimum and maximum values (whiskers), the upper and lower quartiles, and the median. The length of the box represents the interquartile range.

47), and our preliminary data suggest that the stromal WNT5A level is significantly correlated with tumor cell YAP1 level in human PDAC (data not shown). Therefore, stromal WNT5A could possibly contribute to YAP1 activation in tumor cells, given that the exuberant desmoplastic stroma is a defining characteristic of PDAC (2). In this scenario, the tumor/stroma interaction will play an instrumental role in orchestrating heterogeneous YAP1 activation in bulk tumor, which may in turn define the molecular heterogeneity and diverse biological phenotypes of PDAC.

Considering that agents targeting the Hippo/YAP pathway are under development (30), our study showed that the critical role of WNT5A-mediated YAP1 activation in a subset of pancreatic tumors of the squamous subtype provides viable therapeutic targets for this most malignant form of human PDAC.

\section{Methods}

Transgenic mice. For the generation of a tamoxifen-inducible PDAC GEM model, Mist $1^{\text {CreERT2/+ }}$ (48) mice were used for conditional activation of mutant $K r a s^{G 12 D}$ and mutant $\operatorname{Trp}^{2} 3^{R 172 H}$ in the mature pancreas. For Yap1 deletion, these mice were further crossed with Yap $1^{f l / f l}$ mice (49). For the most efficient recombination, tamoxifen was administered intraperitoneally to 6-week-old mice in corn oil once daily for 5 days. The recombination efficiency was tested using PCR primers designed specifically to detect wild-type and recombinant alleles of Kras, Trp53, and Yap1 in pancreatic tissues.

Cell culture and establishment of primary PDAC lines. Human pancreatic cell lines SNU410, HPAC, HPAF-II, PL45, PaTu8988S, and PaTu8988T were obtained from the American Type Culture Collection (ATCC). Pa04C was established from resected patient tumors, maintained as low passage $(<10)$ (50), and cultured according to recommended protocols. Establishment and maintenance of primary 


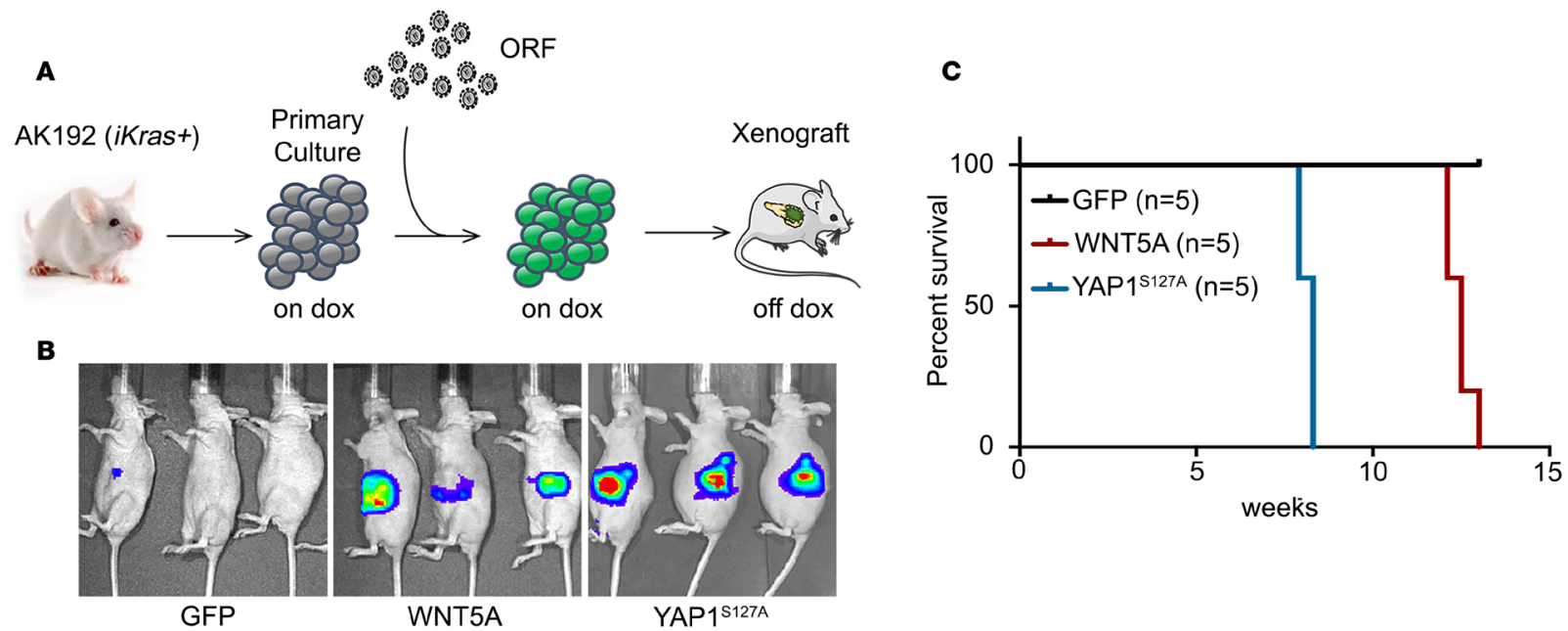

D

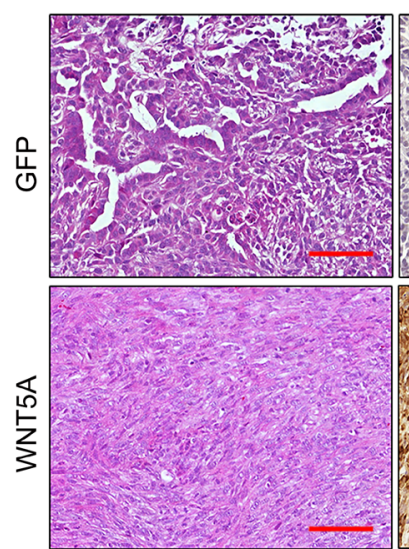

HE

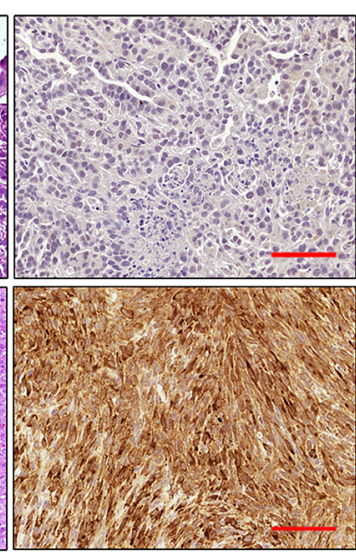

WNT5A

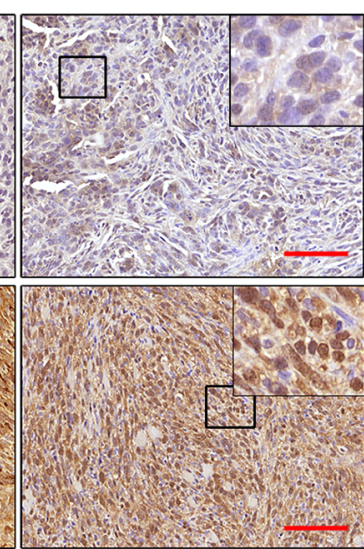

YAP1

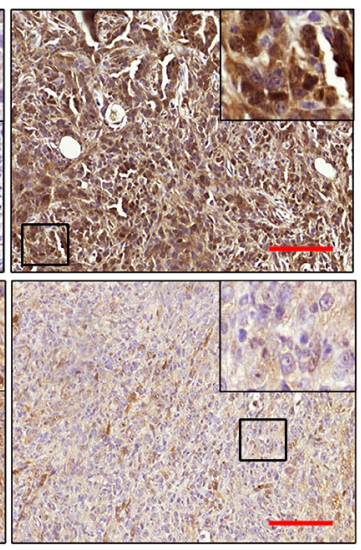

pERK

Figure 6. WNT5A overexpression drives KRAS-independent tumor growth. (A) Schematic workflow for the in vivo KRAS bypass experiment. (B) AK192 cells expressing luciferase were infected with lentivirus expressing GFP, WNT5A, or YAP1 ${ }^{5127 A}$ and orthotopically injected into nude mice pancreas in the presence of doxycycline. Animals were withdrawn from doxycycline 4 days later, and tumor growth was visualized by bioluminescent imaging at 8 weeks. (C) Kaplan-Meier overall survival analysis for nude mice ( $n=5$ /group) orthotopically transplanted with the cells described in B. (D) Orthotopic xenograft tumors generated with AK192-GFP cells (on doxycycline) or AK192-WNT5A cells (off doxycycline) were stained for WNT5A, YAP1, and phospho-ERK. Scale bar: $100 \mu \mathrm{m}$.

mouse PDAC lines were performed as described previously $(8,14)$. Mouse PDAC cell line PD3077 was a gift from Ben Stanger, University of Pennsylvania Perelman School of Medicine (Philadelphia, Pennsylvania, USA). The human patient PDX cell lines were maintained in RPMI-1640 medium containing 10\% FBS (Clontech). KRAS mutation status, molecular subtypes, and tumor grade information are listed in Supplemental Table 9.

Reagents. We used doxycycline (RPI), PE Annexin V Apoptosis Detection Kit I (BD Biosciences), and BOX5 (MilliporeSigma).

Immunostaining and Western blot analysis. Immunohistochemical analysis was performed as described previously (51). Details for immunofluorescence staining, Western blot analysis, and primary antibody information are described in the Supplemental Methods.

Lentivirus-mediated shRNA knockdown. All lentiviral shRNA clones targeting YAP1, WNT5A, and nontargeting shRNA control were obtained from MilliporeSigma in the pLKO vector. The clone IDs for the shRNA are listed in the supplemental materials.

CRISPR/Cas9-mediated gene KO. SgRNAs targeting mouse Wnt5a or Yap 1 were cloned into pSpCas9(BB)-2A-Puro (Addgene, 62988) and transfected into target cells. After $2 \mu \mathrm{g} / \mathrm{mL}$ puromycin selection for 1 week, single-cell clones were isolated and analyzed by T7E1 assay and Western blot analysis. Sequences for Wnt5a and Yap1 sgRNA are listed in supplemental materials. 
A

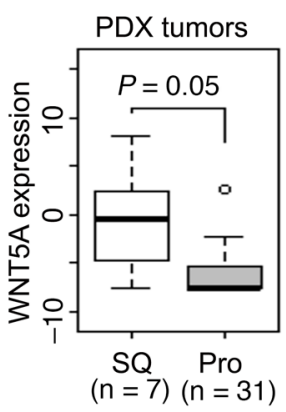

D

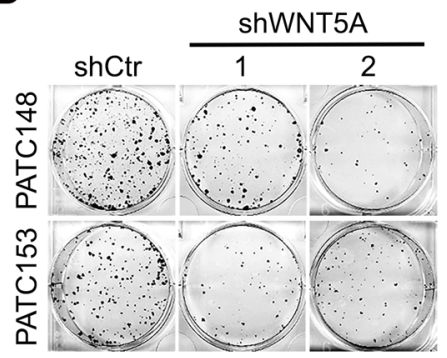

B

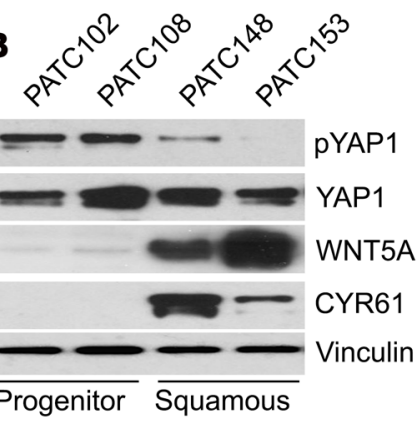

$\mathbf{E}$

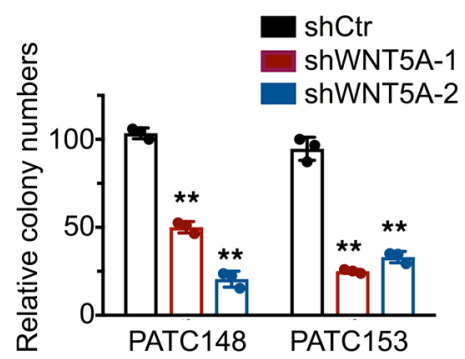

C

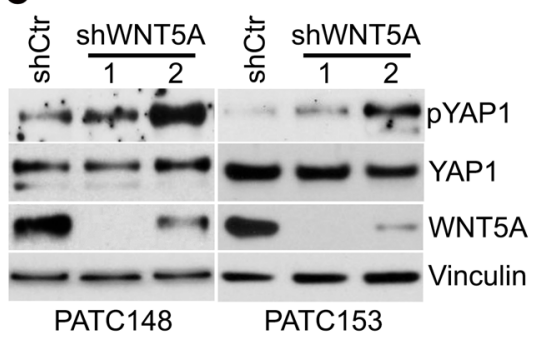

$\mathbf{G}$

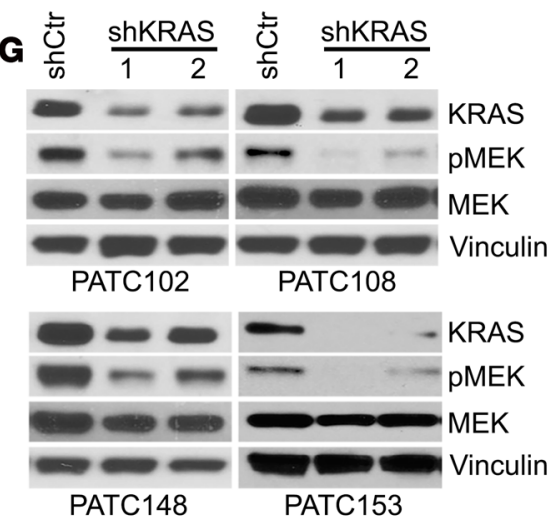

H

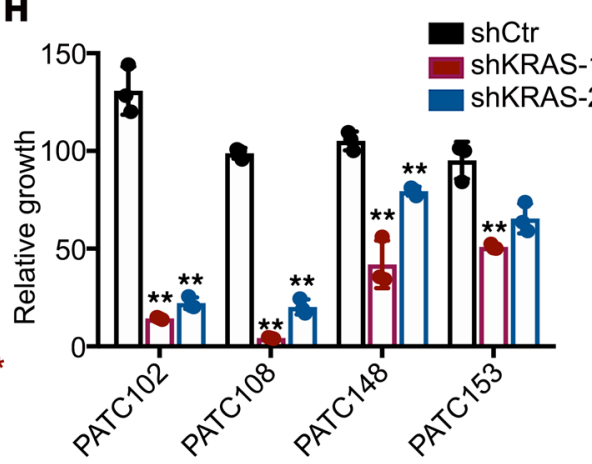

Figure 7. The WNT5A/YAP1 axis is active in primary human PDAC and required for tumor maintenance. (A) WNT5A expression in squamous or progenitor subtype PDXs. (B) Western blot analysis for WNT5A, YAP1, phospho-YAP1, and CYR61 in PDX cell lines of squamous or progenitor subtype. WNT5A blot was run in parallel with the rest of the blots contemporaneously. (C) Western blot analysis for WNT5A, YAP1, and phospho-YAP1 in squamous subtype PDX cell lines infected with WNT5A shRNAs or nontargeting shRNA. The PYAP1 blot for PATC153 was run in parallel with the rest of the blots for PATC153 contemporaneously. (D) Representative images of the colony formation assay for squamous subtype PDX cell lines infected with WNT5A shRNAs or nontargeting shRNA. (E) Quantification from triplicates is shown. (F) PATC108 and PATC148 cells infected with WNT5A shRNAs or nontargeting shRNA were subcutaneously injected into nude mice. Tumor volumes were measured on the indicated dates after injection, and relative tumor growth normalized to day 14 was presented. Results are presented as the means \pm SD $(n=5)$. (C) Western blot analysis for KRAS, phospho-MEK1/2, and MEK1/2 in PDX cell lines infected with KRAS shRNAs or nontargeting shRNA. (H) Cell growth assay for PDX cell lines infected with KRAS shRNAs or nontargeting shRNA. Quantification from triplicates is presented as relative cell growth upon normalization to the shCtr group. $P$ value was corrected with Dunnett's method. The box plots depict the minimum and maximum values (whiskers), the upper and lower quartiles, and the median. The length of the box represents the interquartile range. Error bars from all panels indicate $\pm \mathrm{SD} .{ }^{* *} P<0.01$.

TMA staining and analysis. Immunohistochemical staining for YAP1 was performed on 5- $\mu$ m unstained sections from the TMA blocks, which included 92 (MD Anderson Cancer Center) or 83 (Johns Hopkins University School of Medicine) PDAC samples from patients who underwent surgery up front. Immunohistochemical staining for YAP1 was reviewed by a pathologist. The expression of YAP1 was classified as YAP1-low and YAP1-high by using the median score for total YAP1 expression (nuclear plus cytoplasmic expression) as a cutoff.

Statistics. Tumor volume and tumor-free survival results were analyzed using GraphPad Prism. To assess distributional differences of variance across various test groups, the Mann-Whitney $U$ test was used. 
Other comparisons were performed by using the unpaired 2-tailed $t$ test. For experiments with more than 2 groups, $P$ value was corrected for multiple comparisons using Dunnett's method. A $P$ value less than 0.05 was considered significant. For all figures with error bars, the SD was calculated to indicate the variation with each experiment and data, and values represent mean \pm SD.

Study approval. All animal studies were approved by the MD Anderson Cancer Center Institutional Animal Care and Use Committee under protocol number 00001549. No patient samples were directly used in this study.

\section{Author contributions}

BT, WY, SFB, SC, QW, LY, XZ, and SG performed data collection and interpretation. JY provided statistical and bioinformatics analysis. CZ and SB contributed to animal breeding. QC, CAB, YK, HZ, HW, JBF, $\mathrm{MK}, \mathrm{TPH}$, and AM provided clinical specimens and data analyses on PDX samples. JZ, HW, and AM performed pathology analyses. GFD, DP, and AM provided crucial feedback on the manuscript. WY, SG, and HY drafted the manuscript. WY, SG, and HY conceived, designed, and supervised the study.

\section{Acknowledgments}

We thank the laboratory of Ben Stanger for sharing mouse PDAC cell line PD3077. We would like to thank the Institute for Applied Cancer Science, the Flow Cytometry and Cellular Imaging Core at The University of Texas MD Anderson Cancer Center, and the Veterinary Medicine Department at MD Anderson (Cancer Center Support Grant CA016672). We thank Ronald DePinho, Alan Wang, Mien-Chie Hung, Guocan Wang, Baoli Hu, Xin Zhou, and Jihye Paik for helpful discussions and critical reviews. The research was supported by the Pancreatic Cancer Action Network-American Association for Cancer Research Career Development Award and National Cancer Institute (NCI) grant R01CA214793 to HY, the Pancreatic Cancer Action Network-American Association for Cancer Research Pathway to Leadership Award to WY, the Seed Grant from Hirshberg Foundation for Pancreatic Cancer Research to HY and WY, and NCI P01 grant P01CA117969 to HW, JBF, MK, GFD, AM, and HY.

Address correspondence to: Wantong Yao, Translational Molecular Pathology Department, The University of Texas MD Anderson Cancer Center, 2130 West Holcombe Boulevard, Houston, Texas 77030, USA. Phone: 713.563.4411; Email: wyao2@mdanderson.org. Or to: Sonal Gupta, Department of Pathology, The University of Texas MD Anderson Cancer Center, 6565 MD Anderson Boulevard, Houston, Texas 77030, USA. Phone: 713.745.0831; Email: sgupta8@mdanderson.org. Or to: Haoqiang Ying, The University of Texas MD Anderson Cancer Center, 1515 Holcombe Boulevard, Houston, Texas 77030, USA. Phone: 713.563.3367; Email: hying@mdanderson.org.

1. Rahib L, Smith BD, Aizenberg R, Rosenzweig AB, Fleshman JM, Matrisian LM. Projecting cancer incidence and deaths to 2030: the unexpected burden of thyroid, liver, and pancreas cancers in the United States. Cancer Res. 2014;74(11):2913-2921

2. Ying H, et al. Genetics and biology of pancreatic ductal adenocarcinoma. Genes Dev. 2016;30(4):355-385.

3. Bailey P, et al. Genomic analyses identify molecular subtypes of pancreatic cancer. Nature. 2016;531(7592):47-52.

4. Cancer Genome Atlas Research Network. Integrated genomic characterization of pancreatic ductal adenocarcinoma. Cancer Cell. 2017;32(2):185-203.e13.

5. Collisson EA, et al. Subtypes of pancreatic ductal adenocarcinoma and their differing responses to therapy. Nat Med. 2011;17(4):500-503.

6. Moffitt RA, et al. Virtual microdissection identifies distinct tumor- and stroma-specific subtypes of pancreatic ductal adenocarcinoma. Nat Genet. 2015;47(10):1168-1178.

7. Collins MA, et al. Oncogenic Kras is required for both the initiation and maintenance of pancreatic cancer in mice. J Clin Invest. 2012;122(2):639-653.

8. Ying H, et al. Oncogenic Kras maintains pancreatic tumors through regulation of anabolic glucose metabolism. Cell. 2012;149(3):656-670.

9. Muzumdar MD, et al. Survival of pancreatic cancer cells lacking KRAS function. Nat Commun. 2017;8(1):1090

10. Meng Z, Moroishi T, Guan KL. Mechanisms of Hippo pathway regulation. Genes Dev. 2016;30(1):1-17.

11. Zhao B, et al. Inactivation of YAP oncoprotein by the Hippo pathway is involved in cell contact inhibition and tissue growth control. Genes Dev. 2007;21(21):2747-2761.

12. Gruber R, Panayiotou R, Nye E, Spencer-Dene B, Stamp G, Behrens A. YAP1 and TAZ control pancreatic cancer initiation in mice by direct up-regulation of JAK-STAT3 signaling. Gastroenterology. 2016;151(3):526-539.

13. Zhang W, et al. Downstream of mutant KRAS, the transcription regulator YAP is essential for neoplastic progression to pancreatic ductal adenocarcinoma. Sci Signal. 2014;7(324):ra42.

14. Kapoor A, et al. Yap1 activation enables bypass of oncogenic Kras addiction in pancreatic cancer. Cell. 2014;158(1):185-197.

15. Murakami S, et al. Yes-associated protein mediates immune reprogramming in pancreatic ductal adenocarcinoma. Oncogene. 
2017;36(9):1232-1244.

16. Cordenonsi M, et al. The Hippo transducer TAZ confers cancer stem cell-related traits on breast cancer cells. Cell. 2011;147(4):759-772.

17. Elsässer HP, Lehr U, Agricola B, Kern HF. Establishment and characterisation of 2 cell lines with different grade of differentiation derived from one primary human pancreatic adenocarcinoma. Virchows Arch, B, Cell Pathol. 1992;61(5):295-306.

18. Kim MH, et al. Actin remodeling confers BRAF inhibitor resistance to melanoma cells through YAP/TAZ activation. EMBO J. 2016;35(5):462-478.

19. Lin L, et al. The Hippo effector YAP promotes resistance to RAF- and MEK-targeted cancer therapies. Nat Genet. $2015 ; 47(3): 250-256$.

20. Shao DD, et al. KRAS and YAP1 converge to regulate EMT and tumor survival. Cell. 2014;158(1):171-184

21. Sweet-Cordero A, et al. An oncogenic KRAS2 expression signature identified by cross-species gene-expression analysis. Nat Genet. 2005;37(1):48-55.

22. Barbie DA, et al. Systematic RNA interference reveals that oncogenic KRAS-driven cancers require TBK1. Nature. 2009;462(7269):108-112.

23. Aiello NM, et al. EMT subtype influences epithelial plasticity and mode of cell migration. Dev Cell. 2018;45(6):681-695.e4.

24. Schaefer CF, et al. PID: the Pathway Interaction Database. Nucleic Acids Res. 2009;37(Database issue):D674-D679.

25. Park HW, et al. Alternative Wnt signaling activates YAP/TAZ. Cell. 2015;162(4):780-794.

26. van Amerongen R. Alternative Wnt pathways and receptors. Cold Spring Harb Perspect Biol. 2012;4(10):a007914.

27. Muramatsu T, et al. YAP is a candidate oncogene for esophageal squamous cell carcinoma. Carcinogenesis. 2011;32(3):389-398.

28. Ambrosini G, Adida C, Altieri DC. A novel anti-apoptosis gene, survivin, expressed in cancer and lymphoma. Nat Med. 1997;3(8):917-921.

29. Caldas H, et al. Survivin splice variants regulate the balance between proliferation and cell death. Oncogene. 2005;24(12):1994-2007.

30. Harvey KF, Zhang X, Thomas DM. The Hippo pathway and human cancer. Nat Rev Cancer. 2013;13(4):246-257.

31. Yu FX, Zhao B, Guan KL. Hippo pathway in organ size control, tissue homeostasis, and cancer. Cell. 2015;163(4):811-828.

32. Mueller S, et al. Evolutionary routes and KRAS dosage define pancreatic cancer phenotypes. Nature. 2018;554(7690):62-68.

33. Somerville TDD, et al. TP63-Mediated enhancer reprogramming drives the squamous subtype of pancreatic ductal adenocarcinoma. Cell Rep. 2018;25(7):1741-1755.e7.

34. Saladi SV, et al. ACTL6A is co-amplified with p63 in squamous cell carcinoma to drive Yap activation, regenerative proliferation, and poor prognosis. Cancer Cell. 2017;31(1):35-49.

35. Adams CR, et al. Transcriptional control of subtype switching ensures adaptation and growth of pancreatic cancer. Elife. 2019;8:e45313.

36. Fernandez-LA, et al. YAP1 is amplified and up-regulated in hedgehog-associated medulloblastomas and mediates Sonic hedgehog-driven neural precursor proliferation. Genes Dev. 2009;23(23):2729-2741.

37. Schwartz AL, et al. Phenylmethimazole decreases Toll-like receptor 3 and noncanonical Wnt5a expression in pancreatic cancer and melanoma together with tumor cell growth and migration. Clin Cancer Res. 2009;15(12):4114-4122.

38. Ripka S, et al. WNT5A--target of CUTL1 and potent modulator of tumor cell migration and invasion in pancreatic cancer. Carcinogenesis. 2007;28(6):1178-1187.

39. Gong R, et al. Opposing roles of conventional and novel PKC isoforms in Hippo-YAP pathway regulation. Cell Res. 2015;25(8):985-988.

40. Grzeschik NA, Parsons LM, Allott ML, Harvey KF, Richardson HE. Lgl, aPKC, and Crumbs regulate the Salvador/Warts/ Hippo pathway through 2 distinct mechanisms. Curr Biol. 2010;20(7):573-581.

41. Kim NG, Gumbiner BM. Adhesion to fibronectin regulates Hippo signaling via the FAK-Src-PI3K pathway. J Cell Biol. 2015;210(3):503-515.

42. Li P, Silvis MR, Honaker Y, Lien WH, Arron ST, Vasioukhin V. $\alpha$ E-catenin inhibits a Src-YAP1 oncogenic module that couples tyrosine kinases and the effector of Hippo signaling pathway. Genes Dev. 2016;30(7):798-811.

43. Sudol M. Yes-associated protein (YAP65) is a proline-rich phosphoprotein that binds to the SH3 domain of the Yes proto-oncogene product. Oncogene. 1994;9(8):2145-2152.

44. Zaidi SK, et al. Tyrosine phosphorylation controls Runx2-mediated subnuclear targeting of YAP to repress transcription. EMBO J. 2004;23(4):790-799.

45. Wang MT, et al. K-Ras promotes tumorigenicity through suppression of non-canonical Wnt signaling. Cell. 2015;163(5):1237-1251.

46. Pilarsky C, et al. Activation of Wnt signalling in stroma from pancreatic cancer identified by gene expression profiling. $J$ Cell Mol Med. 2008;12(6B):2823-2835.

47. Seino T, et al. Human pancreatic tumor organoids reveal loss of stem cell niche factor dependence during disease progression. Cell Stem Cell. 2018;22(3):454-467.e6.

48. Habbe N, et al. Spontaneous induction of murine pancreatic intraepithelial neoplasia ( $\mathrm{mPanIN}$ ) by acinar cell targeting of oncogenic Kras in adult mice. Proc Natl Acad Sci USA. 2008;105(48):18913-18918.

49. Cai J, Zhang N, Zheng Y, de Wilde RF, Maitra A, Pan D. The Hippo signaling pathway restricts the oncogenic potential of an intestinal regeneration program. Genes Dev. 2010;24(21):2383-2388.

50. Jones $\mathrm{S}$, et al. Core signaling pathways in human pancreatic cancers revealed by global genomic analyses. Science. 2008;321(5897):1801-1806.

51. Aguirre AJ, et al. Activated Kras and Ink4a/Arf deficiency cooperate to produce metastatic pancreatic ductal adenocarcinoma. Genes Dev. 2003;17(24):3112-3126. 